\title{
LIBROS Y REVISTAS RECIBIDOS
}

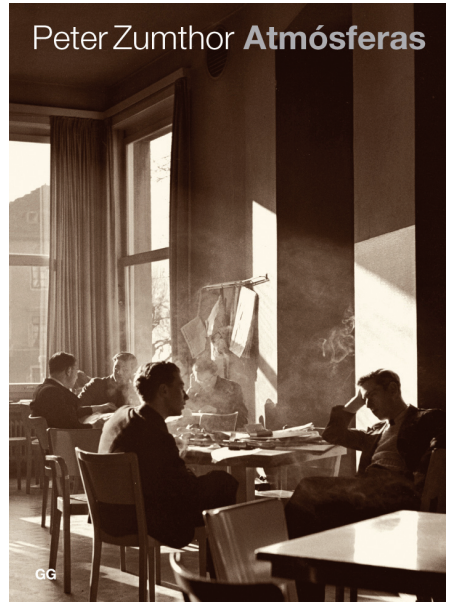

LOS OJOS DE LA PIEL.

LA AROUITECTURA Y LOS SENTIDOS

Juhani Pallasmaa

Editorial Gustavo Gili S.A., Barcelona

80 páginas, $15,5 \times 21,5 \mathrm{~cm}, \mathrm{~b} / \mathrm{n}$

2006

Texto castellano

Web www.ggili.com

"Si hubiera sido más fácil entender el cuerpo, nadie habría pensado que teníamos una mente". R. Rorty. Los estudios de los biólogos han orientado las preocupaciones, no sólo de los filósofos, sociólogos y antropólogos, también la mirada de algunos arquitectos y teóricos de la arquitectura, en otros sentidos que no sea la vista, como el tacto, el oído y el olfato por ejemplo, y hecho reconsiderar arquitecturas antiguas y nuevas que los han tenido presentes.

Cuando un lugar es acogedor habría que pensar, por qué. Las obras actuales de Glenn Murcutt, Steven Holl o Peter Zumthor se relacionan con experiencias sensoriales complejas.

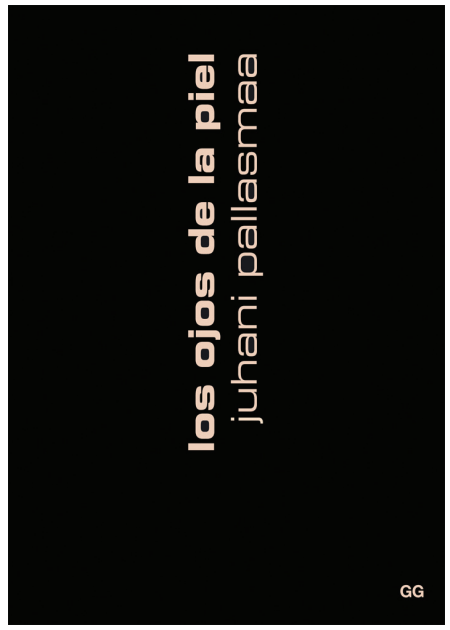

\section{Atmósferas}

Peter Zumthor

Editorial Gustavo Gili S.A., Barcelona

76 páginas, $17 \times 23,5 \mathrm{~cm}$, color

2006

Texto castellano

Web www.ggili.com

El texto es de una charla dada por Zumthor y parte diciendo: "hace ya mucho tiempo que me interesa qué es la calidad propiamente arquitectónica". Y se contesta: "para mí la realidad arquitectónica sólo puede tratarse de que un edificio me conmueva o no".

Para ir precisando más se va refiriendo a una serie de magníficas fotos, que desde la de la portada nos sorprenden con su atmósfera y suntuosidad sin pretensiones y con ejemplos de músicos como Brahms, Stravinski, etc. Ya que la música, dice, es la más grande de las artes. Una conferencia inolvidable.

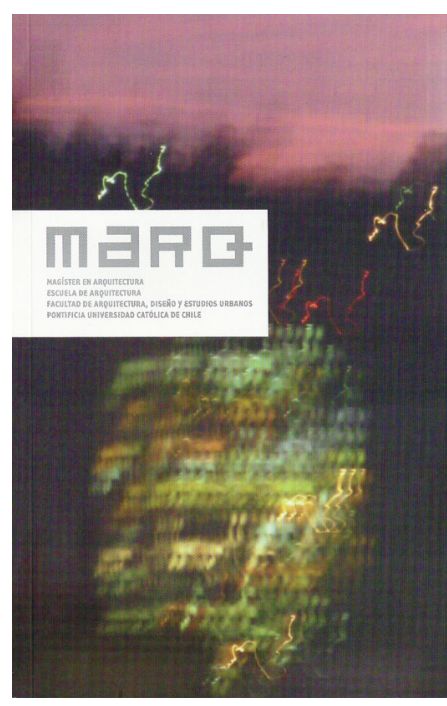

\section{MARQ N 02}

Horacio Torrent, director editorial Programa Magíster en arquitectura FADEU PUC

Anual

102 páginas, $13,5 \times 21 \mathrm{~cm}$, color

Marzo de 2007

Texto castellano

Web www.magisterarq.cl

La introducción del decano José Rosas caracteriza que la comprensión profunda del problema a resolver en el magíster es superar los modos ortodoxos de organización, control y producción de manera que el proyecto sea imprevisible, en el sentido de hallazgo, enriquecimiento de la arquitectura y de la sociedad.

Esto parece cumplirse al revisar los estudios y propuestas urbanas de la periferia de Santiago, la ordenación del vacío entre Cerrillos y el cerro de Chena hasta la escala mínima de la Hydroponic wall house y un soporte publicitario.

\section{Arquitectura y CONStrucción. ChILE 1945-1950. \\ UNA REVISTA DE ARQUITECTURA MODERNA}

Hugo Mondragón, Andrés Téllez Universidad Central de Chile. Facultad de Arquitectura, Urbanismo y Paisaje. 290 páginas, $19,5 \times 24,5 \mathrm{~cm}, \mathrm{~b} / \mathrm{n}$

2006

Texto castellano

Web www.ucentral.cl

Lo que ocurrió en la arquitectura y el urbanismo en Chile entre 1945 y 1950 es sorprendente y está resumido en esta revista, cuyos 18 números analiza esta publicación en 8 interesantes capítulos ilustrados, finalizando con una entrevista al arquitecto y profesor Euclides Guzmán, muy aclaratoria. Como los autores comentan, el movimiento moderno tuvo diferentes interpretaciones que diferencian lo que ocurrió en Brasil, Argentina y Chile por ejemplo, donde hay obras en vivienda social, edificios, casas unifamiliares y proyectos urbanos que atestiguan hasta hoy su gran calidad arquitectónica y adecuación al uso.

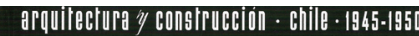

Una peylsta de apruttectura modera

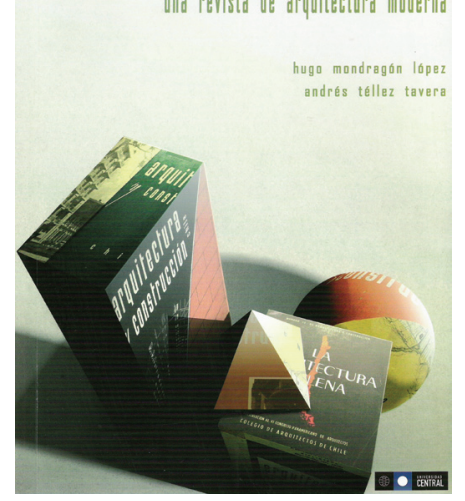

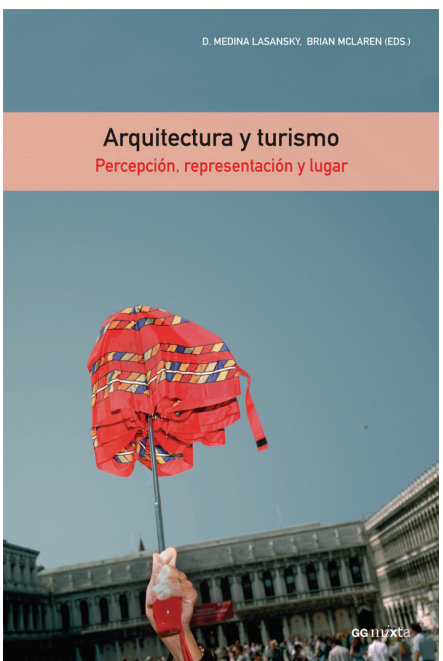

ArQuitectura Y tURISMO.

Percepción, Representación y lugar

D. Medina Lasansky, Brian McLaren, editores

Editorial Gustavo Gili S.A., Barcelona 320 páginas, $15 \times 22,5 \mathrm{~cm}, \mathrm{~b} / \mathrm{n}$

2006

Texto castellano

Web www.ggili.com

Trece autores que profundizan en causas y efectos de una actividad en expansión con disciplinas como la antropología, la geografía cultural, la economía, ciencias políticas y sociología en lo que se revela como muy ligado a causas y efectos en la arquitectura.

Los autores se refieren $a$, por ejemplo, los turistas británicos en Italia en la segunda mitad del s. XIX, el Pueblo Español, recinto en la Exposición Universal de Barcelona del año 1929 y el manejo político del dictador Primo de Rivera y del rey Alfonso XIII, La nueva política del espectáculo: "Bilbao" y la imaginación local y llega al Luxor Las Vegas, un ensayo notable. Recomendado para arquitectos que intentan averiguar los por qué. 


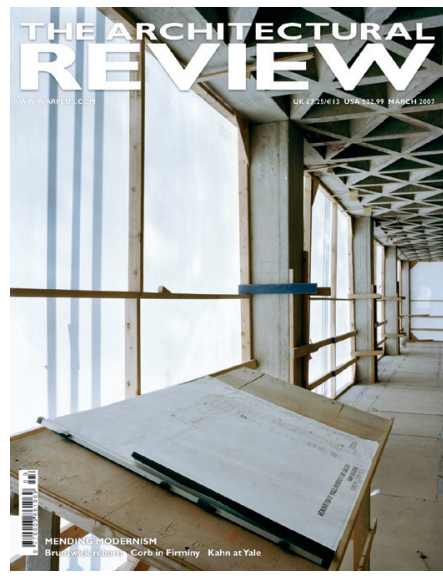

The Architectural Review № 1321 Mending MOdeRnISM

Paul Finch, director

Emap Communications, Londres

Mensual

98 páginas, $23 \times 29,5 \mathrm{~cm}$, color

Marzo de 2007

Texto inglés

Web www.arplus.com

Un número sobre mending modernism con obras desde 1949 hasta sus remodelaciones, o demoliciones de los años 80 y 90 Deriva de una serie de conferencias organizadas por la revista en diciembre 2006.

Un material bien expuesto, incluye la construcción de la iglesia de Firminy cuarenta años después.

El interés es reconsiderar estas obras dentro de la ciudad y sus mutuas relaciones: el eclipse de Brunswick, el resurgimiento de Yale, la galería de Kahn, el Barbican Centre de Londres, una academia de Arte en Holanda y Plymouth, destruida por la Segunda Guerra y reconstruida entre 1949 y 1952.

Un número indispensable si queremos hacer algo por Santiago.
Quaderns d'arquitectura I URBANISME $\mathbf{N}^{\circ} \mathbf{2 5 1}$

Oriol Bohigas, redactor editorial Collegi d'Arquitectes de Catalunya, Barcelona

Trimestral

160 páginas, $24 \times 31 \mathrm{~cm}$, color Otoño 2006

Texto catalán / inglés / castellano

Web dgr@coac.net

El contenido es tan variado y poderoso como en las dos anteriores revistas. Las distintas secciones: proyecto, doméstica urbanismo, construcción, arquitectura histórica, entrevista, lecturas, política, escuelas de arquitectura, concursos, etc., demuestran una capacidad para abordar en profundidad todos los temas que la profesión trae y además, si es necesario, transcribir un poema iracundo de Jorge Oteiza contra los catalanes, junto a observaciones muy precisas y finas como recordar una frase de Alvar Aalto "como si las obras fueran siempre reformas" o de la bióloga Premio Nobel italiana, Rita Levi, en su autobiografía Elogio de la imperfección acerca de las cosas inacabadas e imperfectas. Una revista que se agradece.
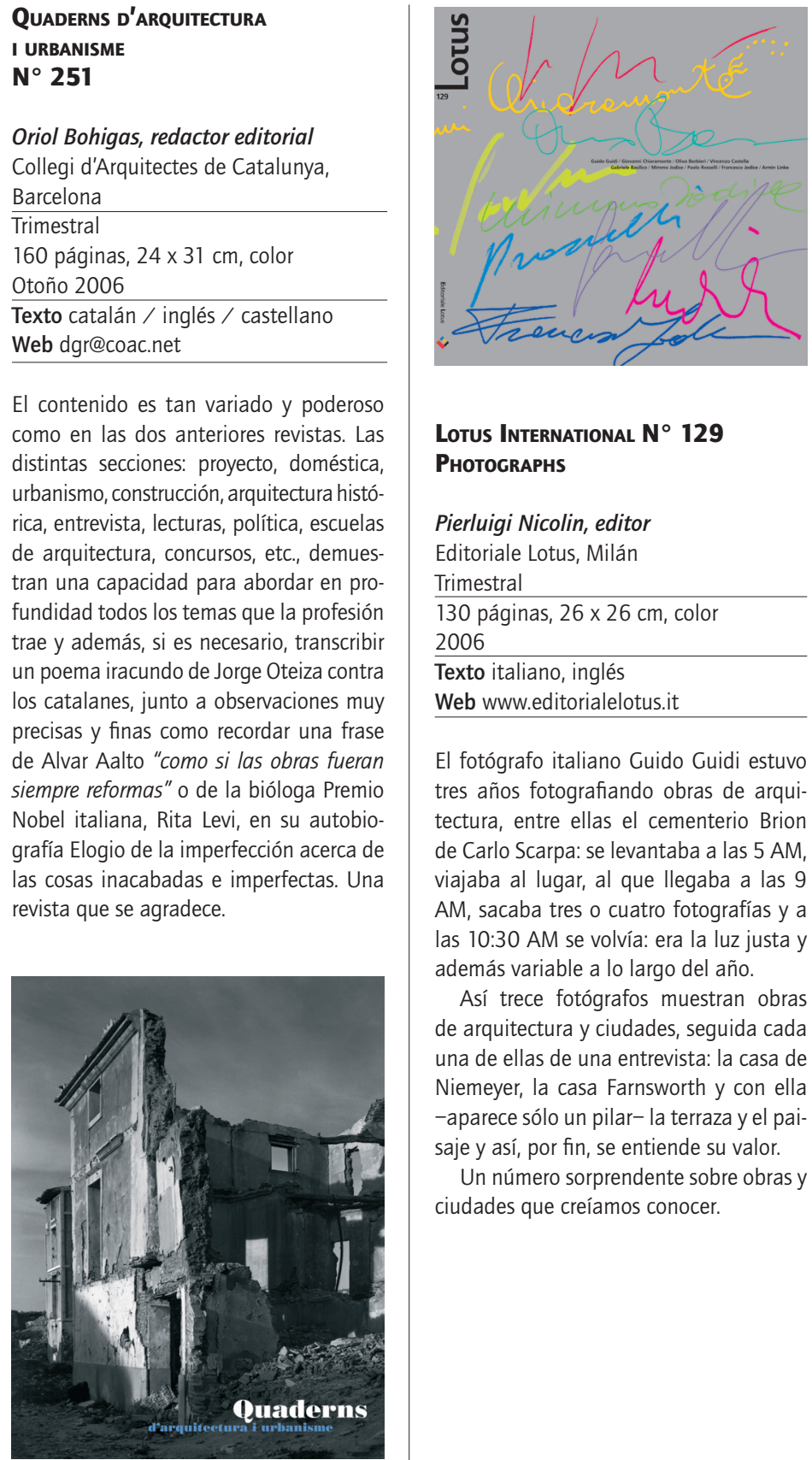

\section{Lotus International $\mathbf{N}^{\circ} 129$}

Photographs

Pierluigi Nicolin, editor

Editoriale Lotus, Milán

Trimestral

130 páginas, $26 \times 26 \mathrm{~cm}$, color

2006

Texto italiano, inglés

Web www.editorialelotus.it

El fotógrafo italiano Guido Guidi estuvo tres años fotografiando obras de arquitectura, entre ellas el cementerio Brion de Carlo Scarpa: se levantaba a las 5 AM, viajaba al lugar, al que llegaba a las 9 AM, sacaba tres o cuatro fotografías y a las 10:30 AM se volvía: era la luz justa y además variable a lo largo del año.

Así trece fotógrafos muestran obras de arquitectura y ciudades, seguida cada una de ellas de una entrevista: la casa de Niemeyer, la casa Farnsworth y con ella - aparece sólo un pilar- la terraza y el paisaje y así, por fin, se entiende su valor.

Un número sorprendente sobre obras y ciudades que creíamos conocer.

\section{Summa+ N 85 Reciclaje} MONTREAL

Fernando Diez, director editorial Donn S.A., Buenos Aires

Bimestral

128 páginas (más 28 de avisos),

$24,5 \times 34 \mathrm{~cm}$, colo

Febrero de 2007

Texto castellano

Web www.summamas.com

Doce ejemplos de reciclaje en Argentina especialmente en Buenos Aires, en edificios de las primeras décadas del s. XX, que dan para sacar algunas conclusiones y plantear algunas preguntas generales que no se hacen: cada proyecto queda encerrado en sí mismo, con largas explicaciones entusiastas de cada autor. Una oportunidad desperdiciada.

Hay una Montreal re-visitada desde la década del 60 hasta hoy y luego, quizás la parte más interesante, una serie de tema diversos: varios muy buenos proyectos expuestos en el Centro de Arquitectura de Viena, AZ W, centrado en estética y compromiso social y una exposición itinerante de libros editados en España entre el 2000 y 2005, una excelente instalación del diseñador catalán Enric Satué en Buenos Aires.

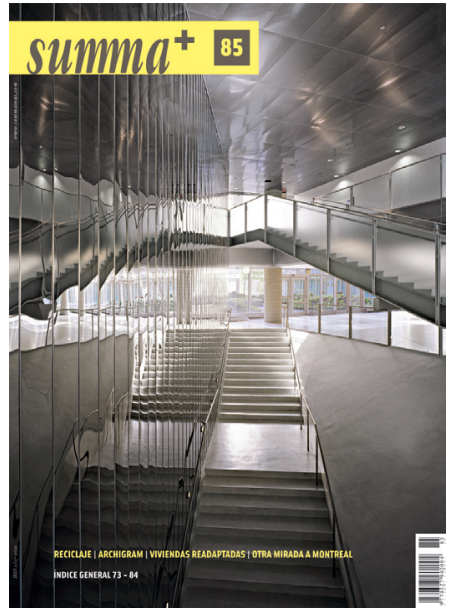

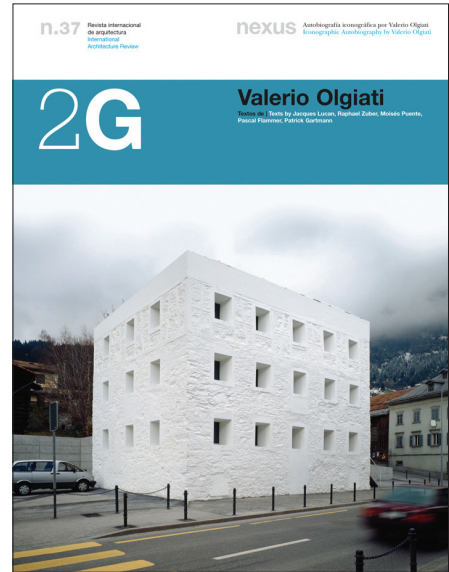

\section{$2 \mathrm{G} \mathbf{N}^{\circ} 37$ Valerio Olgiati}

Mónica Gili, directora

Editorial Gustavo Gili S.A., Barcelona

Trimestral

144 páginas, $23 \times 30 \mathrm{~cm}$, color

2006

Texto castellano, inglés

Web www.ggili.com

Este arquitecto suizo de 49 años tiene ya una obra variada en temas y tamaño de encargos, en diferentes países, la que aborda con una contención y precisión extraordinarias, que quizás por eso mismo emocionan, como la buena poesía. Sus asesores más importantes son un ingeniero civil que estudió luego arquitectura, Patrick Gartmann y Peter Diggelmann, carpintero, arquitecto y capataz de obra.

La revisión de la obra de Olgiati desde una casa (1989-1991) hasta el proyecto del Museo de Taipei en Taiwán. 